\title{
Reduction of autofluorescence in whole adult worms of Schistosoma japonicum for immunofluorescence assay
}

\author{
Qunfeng $\mathrm{Wu}^{1}$, Zheng Feng ${ }^{2}$ and Wei $\mathrm{Hu}^{1,2^{*}}$
}

\begin{abstract}
Immunofluorescence assay is one of methods used to understand spatial biology by visualizing the localization of biomolecules in cells and tissues. Autofluorescence, as a common phenomenon in organisms, is a background signal that interferes with the immunolocalization assay of schistosome biomolecules, and may lead to misinterpretation of the biomolecular function. However, an effective method for reducing the autofluorescence in Schistosoma remains unclear. In order to find a suitable method, different chemical reagents including Sudan black B (SBB), trypan blue (TB), copper sulfate (CuSO$)_{4}$, Tris-glycine (Gly), and ammonia/ethanol (AE) were tested at different concentrations and treatment times, and $\mathrm{SBB}$ and $\mathrm{CuSO}_{4}$ were verified for the effect of blocking autofluorescence in immunofluorescence to localize the target with anti-SjCRT antibody. By comparing the autofluorescence characteristics of different conditions, it was found that $\mathrm{SBB}, \mathrm{TB}$, and $\mathrm{CuSO}_{4}$ were effective in reducing the autofluorescence effect to various degrees, with the best effects produced with $50 \mathrm{mM} \mathrm{CuSO}_{4}$ for $6 \mathrm{~h}$ and $0.5 \% \mathrm{SBB}$ for $6 \mathrm{~h}$ in females and males, respectively. We then applied the optimized conditions to the immunofluorescence of the SjCRT protein, and the results revealed that the immunofluorescence signal of SjCRT was clearly visible without autofluorescence interference. Therefore, we present an effective method for reducing autofluorescence in male and female worms of Schistosoma japonicum for immunofluorescence assay, which could be helpful to better understand biomolecular functions. Our method provides a foundation for immunofluorescence assay in other flukes with autofluorescence.
\end{abstract}

Keywords: Schistosoma japonicum, Reducing autofluorescence, Immunofluorescence, Sudan black B, Copper sulfate

Schistosomiasis is a neglected tropical disease caused by trematodes of the genus Schistosoma, posing a great threat to the health of humans and animals, and impacting economic development [1]. Immunofluorescence assay (IFA) has been widely applied in a host of biological studies to visualize the spatial location of target molecules of interest in a great variety of different types of

\footnotetext{
*Correspondence: huw@fudan.edu.cn

${ }^{1}$ State Key Laboratory of Genetic Engineering, Ministry of Education Key Laboratory of Contemporary Anthropology, Collaborative Innovation Center for Genetics and Development, School of Life Sciences, Fudan University, Shanghai 200438, People's Republic of China

Full list of author information is available at the end of the article
}

tissue and cell preparations [2, 3]. IFA is also an essential tool for identifying and localizing schistosome molecules of interest to better understand the worm developmental strategy and parasite-host molecular interplay [4-6]. The assay capability is achieved by using fluorophore-labeled antibodies to directly or indirectly react with a target antigen in biological samples. However, this technique is disadvantaged by unwanted fluorescence due to autofluorescence (AF) from either the target tissue or the fluorescent background caused by non-specific binding of the fluorescent label. AF interference is one of the shortcomings of IFA, particularly when using confocal laser scanning microscopy (CLSM), which requires minimal tissue $\mathrm{AF}$ and a reduced non-specific fluorescence background. original author(s) and the source, provide a link to the Creative Commons licence, and indicate if changes were made. The images or other third party material in this article are included in the article's Creative Commons licence, unless indicated otherwise in a credit line to the material. If material is not included in the article's Creative Commons licence and your intended use is not permitted by statutory regulation or exceeds the permitted use, you will need to obtain permission directly from the copyright holder. To view a copy of this licence, visit http://creativecommons.org/licenses/by/4.0/. The Creative Commons Public Domain Dedication waiver (http://creativecommons.org/publicdomain/zero/1.0/) applies to the data made available in this article, unless otherwise stated in a credit line to the data. 
AF, either intrinsic or induced by fixation processing, may either mask specific fluorescence signals or be mistaken for fluorescent labels [7].

Biological AF, emitted from endogenous fluorophores, is an intrinsic property of cells and tissues, commonly seen in mammals $[8,9]$, rodents [10], nematodes [11], and Schistosoma [12]. It is also noted that the AF properties of specific tissue constituents may be of diagnostic value or indicative of cell viability. In schistosomes, the AF of eggs has been used to detect eggs in diseased tissues [13]. AF of the vitelline gland in female schistosomes is emitted mainly from vitelline cells, which can be used to separate and enrich vitelline cells [14] or applied for vitelline gland localization [15]. However, AF is often a noise signal in IFA, and therefore has been a significant concern.

Various histochemical techniques for blocking AF have been evolving. Sudan black B (SBB), trypan blue (TB), copper sulfate $\left(\mathrm{CuSO}_{4}\right)$, Tris-glycine (Gly), and ammonia/ethanol (AE) have been used to control AF [16-18]. The efficacy of chemical reagents in reducing AF differs with the sample type. It was reported that $\mathrm{CuSO}_{4}$ was used to quench AF within the vitellarium of Schistosoma mansoni [19], but little is known of applicable reagents for reducing the AF in S. japonicum.

The present study characterized the AF of male and female $S$. japonicum, and tested five chemical reagents to assess their efficacy in reducing the AF in IFA.

Parasites and animals were prepared. Female Kunming mice (20-25 g) (Shanghai Animal Center, Chinese Academy of Sciences, China) were infected with $80 \pm 5$ S. japonicum cercariae (provided by the National Institute of Parasitic Diseases, China Center for Disease Control and Prevention). Adult worms were harvested by perfusion with ice-cold $0.9 \% \mathrm{NaCl}$ solution containing heparin $(10 \mathrm{U} / \mathrm{mL})$ (Sangon Bioengineering Technical Services, China) at 28 days post-infection. Male and female worms were gently separated, fixed with $4 \%$ paraformaldehyde (Sangon Bioengineering Technical Services, China) for $2 \mathrm{~h}$ at room temperature, and then kept overnight at $4{ }^{\circ} \mathrm{C}$.
After fixation, the worms were treated with $1 \%$ sodium dodecyl sulfate (SDS) in phosphate-buffered saline (PBS) for 20 min. A blocking solution (2\% goat serum, 1\% skim milk powder, $0.1 \%$ cold fish skin gelatin, $0.1 \%$ Triton $\mathrm{X}-100,0.05 \%$ Tween 20, 0.05\% NaN3 in PBS) was applied at $4{ }^{\circ} \mathrm{C}$ overnight. Worms were washed three times with PBS.

To examine the AF of $S$. japonicum, the fixed male and female worms were mounted on slides with $80 \%$ glycerol (Sinopharm Chemical Reagent Co., China) and viewed using CLSM (Nikon A1R, Nikon Instruments, Japan). The fluorescence signals were aligned to four fluorescence channels with the following filter settings of excitation and emission wavelength: DAPI, 405-450 nm; EGFP, 488-515 nm; mCherry, 561-610 nm; and Alexa Fluor 647 (AF647), 640-665 nm (Table 1).

To ascertain the effectiveness of different reagents in controlling the AF arising from schistosomes, we tested five chemical reagents (Sigma-Aldrich), including $\mathrm{CuSO}_{4}$, $\mathrm{SBB}, \mathrm{TB}, \mathrm{Gly}$, and $\mathrm{AE}$, at different concentrations and for different treatment times. The whole worms were immersed in $\mathrm{CuSO}_{4}$ at $0.5,5$, or $50 \mathrm{mM}$ in $50 \mathrm{mM}$ ammonia acetate for $1.5,3$, or $6 \mathrm{~h}$; in $0.01 \%, 0.1 \%$, or $0.5 \% \mathrm{SBB}$ in $70 \%$ ethanol for 1,2 , or $6 \mathrm{~h}$; with $0.05 \%$ TB for 1 or $2 \mathrm{~h}$; with $0.1 \mathrm{M}$ Gly in TBS (pH 7.4) for $2 \mathrm{~h}$; and in $0.25 \%$ ammonia in $70 \%$ ethanol for $2 \mathrm{~h}$. All procedures were performed at room temperature. To remove the excess testing regents, the worms were washed six times for 20 min each with $0.02 \%$ Tween 20 in PBS (PBST). The worms were then placed on a slide, mounted with $80 \%$ glycerol, and viewed with CLSM.

To verify the reactivity of the anti-SjCRT antibodies with crude antigens of $S$. japonicum worms, western blotting (WB) was performed. The worm protein was extracted with 10-20 worms in $1 \mathrm{ml}$ PBS by sonication on ice and then centrifuged for $10 \mathrm{~min}$ at $13,000 \times g$ at $4{ }^{\circ} \mathrm{C}$. WB was performed as described previously [20]. The protein extracts ( $50 \mu \mathrm{g}$ protein) of adult female and male worms were resolved by $12 \%$ SDS-polyacrylamide gel electrophoresis (PAGE) and electrotransferred onto a polyvinylidene fluoride membrane. The membrane was

Table 1 Fluorescence image acquisition using CLSM

\begin{tabular}{lllll}
\hline Acquisition channel & Fluorophore used & Excitation $(\mathrm{nm})$ & Emission $(\mathrm{nm})$ & Image display color \\
\hline DAPI & DAPI & 405 & 450 & Blue \\
EGFP & na & 488 & 515 & Green \\
Cy3 & Cy3 & 532 & 590 & Red \\
mCherry & na & 561 & 610 & Red \\
AF647 & na & 640 & 665 & Purple
\end{tabular}

DAPI 4',6-diamidino-2-phenylindole, Cy3 cyanine3, AF647 Alexa Fluor 647, na not applicable 
incubated with blocking solution (PBS, $\mathrm{pH}$ 8.0, $0.05 \%$ Tween $20,5 \%$ skim milk) at $4{ }^{\circ} \mathrm{C}$ overnight. The membrane was then washed three times with PBST (PBS with $0.1 \%$ Tween 20), and rabbit anti-SjCRT (S. japonicum calreticulin) immunoglobulin G (IgG) (Shanghai YouKe Biotechnology, China) diluted at 1:2000 in PBST was applied for $6 \mathrm{~h}$ at room temperature. The blot was then incubated in a blocking solution containing horseradish peroxidase (HRP)-conjugated goat anti-rabbit IgG (BBI, Shanghai, China) at a dilution of 1:8000 for $2 \mathrm{~h}$ at room temperature. After washing with PBST, the membrane was developed with NcmECL Ultra solution (NCM Biotech, Suzhou, China) and imaged using the Tanon 5200 imaging system (Tanon, Shanghai, China). The skim milk powder, Tween 20, and Triton X-100 were from Sangon Bioengineering Technical Services, China; goat serum was from Zhejiang Tianhang Biotechnology, China; all other reagents were purchased from Sigma-Aldrich.

IFA was performed as described in a previous report [15]. After fixation, blocking of non-specific antigens, and treatment with the optimal conditions to control AF, the worms were incubated with rabbit anti-SjCRT IgG (1:300-500) in blocking solution at $4{ }^{\circ} \mathrm{C}$ for 3 days, followed by washing three times for $2 \mathrm{~h}$ each with PBST. For fluorescence staining, the worms were incubated with Cy3-labeled goat anti-rabbit IgG (1:500) in blocking solution at $4{ }^{\circ} \mathrm{C}$ for 3 days, and at the end of the second day, DAPI staining solution (1:50) was added to the incubation solution. After washing three times for $2 \mathrm{~h}$ each with PBST, the worms were placed on a slide, mounted with $80 \%$ glycerol, and viewed using CLSM (Nikon A1R, Nikon Instruments Inc., Japan). In IFA, DAPI and Cy3 fluorescent dyes were used for labeling nuclear and SjCRT protein, respectively $[21,22]$, and the fluorescence signals were detected in DAPI and Cy3 channels, respectively. The EGFP detection channel was used for monitoring green $\mathrm{AF}$ and evaluating the $\mathrm{AF}$ reduction effect. The fluorescence images of whole-mount worms were examined using CLSM. The DAPI, Cy3, and EGFP channels of microscopy conditions for fluorescence imaging are shown in Table 1. The DAPI was purchased from Boster Biological Technology, China. The Cy3-labeled goat anti-rabbit IgG was from Beyotime Biotechnology, China.

After preparative processing, the worms were mounted with $80 \%$ glycerol and placed under a confocal laser microscope for observation. It was observed that both female and male worms had AF in four different channels (DAPI, EGFP, mCherry, and AF647). In female worms, AF was seen in the tegument, vitelline gland, ovary, eggs, and the worm head (Fig. 1a). However, no blue AF was observed in the vitelline gland, ovary, or eggs (Fig. 1a DAPI column). AF in male schistosomes was mainly distributed in the intestine, tegument, and gynecophoric canal (Fig. 1b). In males, the fluorescence intensity at the intestine was stronger. In male and female worms, the strongest intensity of AF was seen under the EGFP channel compared to others (Fig. 1).

Five different chemical reagents were used to treat male and female worms for reduction of $\mathrm{AF}$, and it was found that the reduction effect varied among reagents, with Gly and $\mathrm{AE}$ having no obvious effects (Additional file 1: Figure $\mathrm{S} 1$ ), while $\mathrm{CuSO}_{4}, \mathrm{SBB}$, and TB had some effect (Fig. 2). It was noted that the effect of blocking AF also differed with the worm sex and the duration of chemical exposure. After treatment with $\mathrm{CuSO}_{4}$, the $\mathrm{AF}$ in both male and female worms was reduced, although the reduction was more significant in female worms (Fig. 2b and c). In females treated with $5 \mathrm{mM} \mathrm{CuSO}_{4}$ for $1.5 \mathrm{~h}$, the AF was attenuated in all four tested channels (Fig. 2b), while treatment for $3 \mathrm{~h}$ led to no detectable AF in mCherry and AF647 channels (Fig. 2c). There was no significant reduction in $\mathrm{AF}$ in the four channels in males treated with $5 \mathrm{mM}$ of $\mathrm{CuSO}_{4}$ for $1.5 \mathrm{~h}$ (Fig. 2b), whereas a reduction was seen in mCherry and AF647 channels after $3 \mathrm{~h}$ of treatment (Fig. 2c).

SBB exhibited some effect in controlling AF in both males and females, particularly in males (Fig. $2 \mathrm{~d}$ and e). In females treated with $0.1 \% \mathrm{SBB}$ for $2 \mathrm{~h}$, AF was reduced in the mCherry channel and blocked in the AF647 channel (Fig. 2e), but treatment with $0.1 \%$ SBB for $1 \mathrm{~h}$ did not exert a perceptible AF reduction effect in females (Fig. 2d). Interestingly, incubation with $0.1 \%$ SBB for $1 \mathrm{~h}$ significantly attenuated AF in male worms in the EGFP and mCherry channels (Fig. 2d); the AF intensity in males in DAPI and EGFP channels was significantly reduced after treatment for $2 \mathrm{~h}$, while it was blocked in the mCherry and AF647 channels (Fig. 2e). $\mathrm{TB}$ showed certain reductive effects on AF in male and female worms, but only in the AF647 channel (Fig. 2f and g).

It was demonstrated that $\mathrm{CuSO}_{4}$ presented better reduction effects on $\mathrm{AF}$ in female worms and $\mathrm{SBB}$ in male worms, and longer time exposure to reagents enhanced the reduction of AF. Different concentrations of $\mathrm{CuSO}_{4}$ and SBB were used to treat worms, and the results are shown in Fig. 3. Compared with the control, all the defined concentrations of $\mathrm{CuSO}_{4}$ and $\mathrm{SBB}$ had AF reduction effects. The AF reduction effects in female worms were increased with increasing $\mathrm{CuSO}_{4}$ concentration, with the AF detected in the EGFP channel more difficult to remove than that in other channels. The effect of AF in male worms was not significantly different between $0.01 \%$ SBB and $0.1 \%$ SBB, but the effect of $0.5 \%$ SBB treatment was significantly better. Among all the chemical solutions, $50 \mathrm{mM} \mathrm{CuSO}_{4}$ showed the best AF reduction 


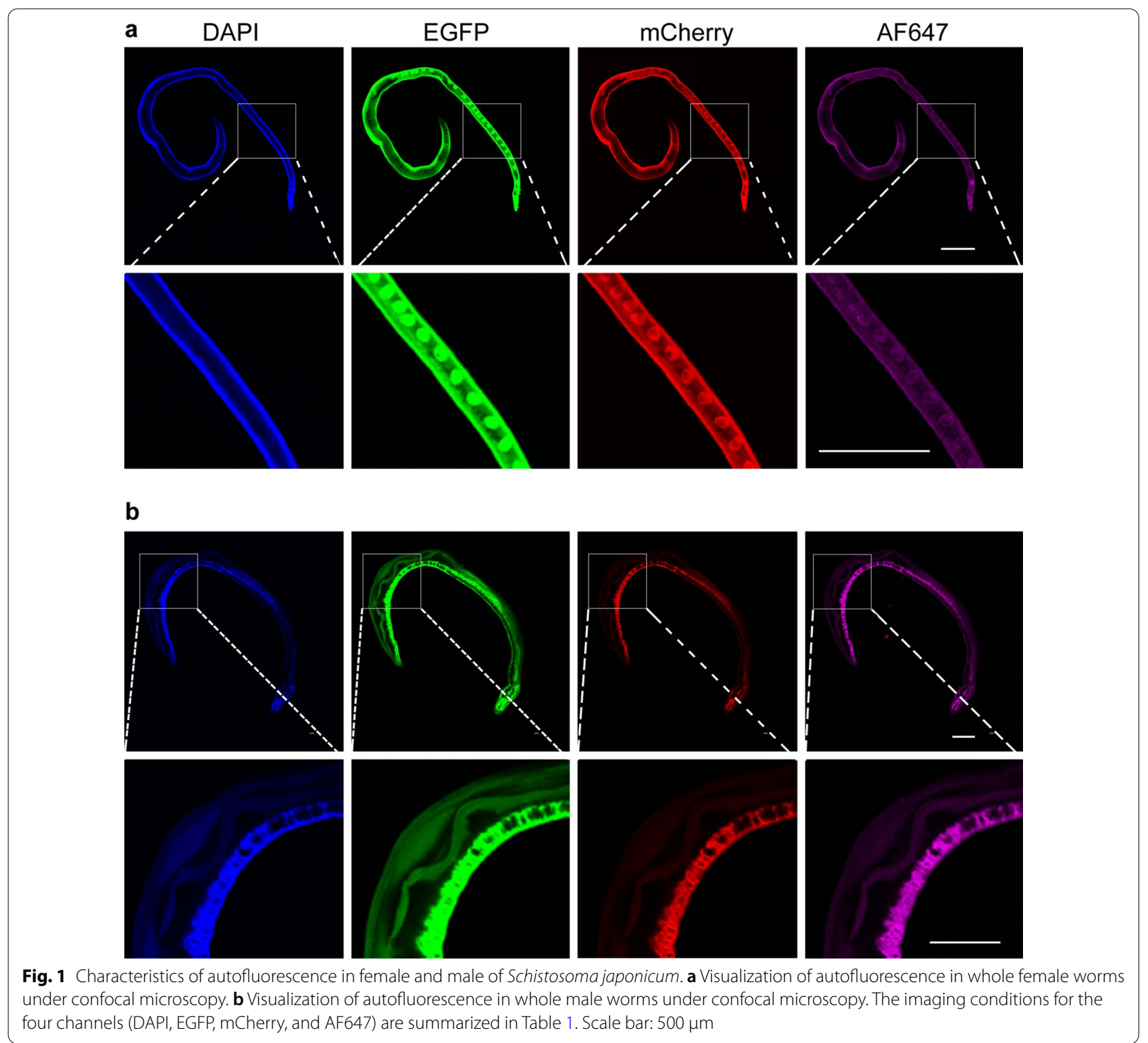

effect in female worms (Fig. 3a), while $0.5 \%$ SBB had the best $\mathrm{AF}$ reduction effect in male worms (Fig. $3 \mathrm{~b}$ ).

WB was used to verify the reactivity of the anti-SjCRT antibodies with crude antigens of male and female worms. The results showed a single band in the crude antigen lanes of both male and female worms, with molecular weight of around $55 \mathrm{kDa}$ (Additional file 2: Figure S2). The band of the female protein lane was less intense than that of the male protein lane (Additional file 2: Figure S2).

In order to verify the effect of AF reduction conditions applied in immunofluorescence experiments, we performed immunofluorescence localization assay on SjCRT. In this assay, fluorescent dye Cy3-labeled goat anti-rabbit IgG was used to localize SjCRT; the nuclei were stained by DAPI. In addition to setting up a normal rabbit serum control, we also monitored the AF using EGFP channels unoccupied by any fluorescent dye. With the treatment for AF reduction in IFA, no significant AF signal was seen in either females or males under the EGFP channel (Fig. 4a and b, Additional file 3: Figure S3). In the normal rabbit serum control, no non-specific immunofluorescence signal was detected in females, but a weak non-specific immunofluorescence signal was seen in males under the $\mathrm{Cy} 3$ channel (Additional file 3: Figure S3). The nuclear staining and SjCRT IF staining signals were scattered and granular in both females and males (Fig. 4), and the weak non-specific immunofluorescence 


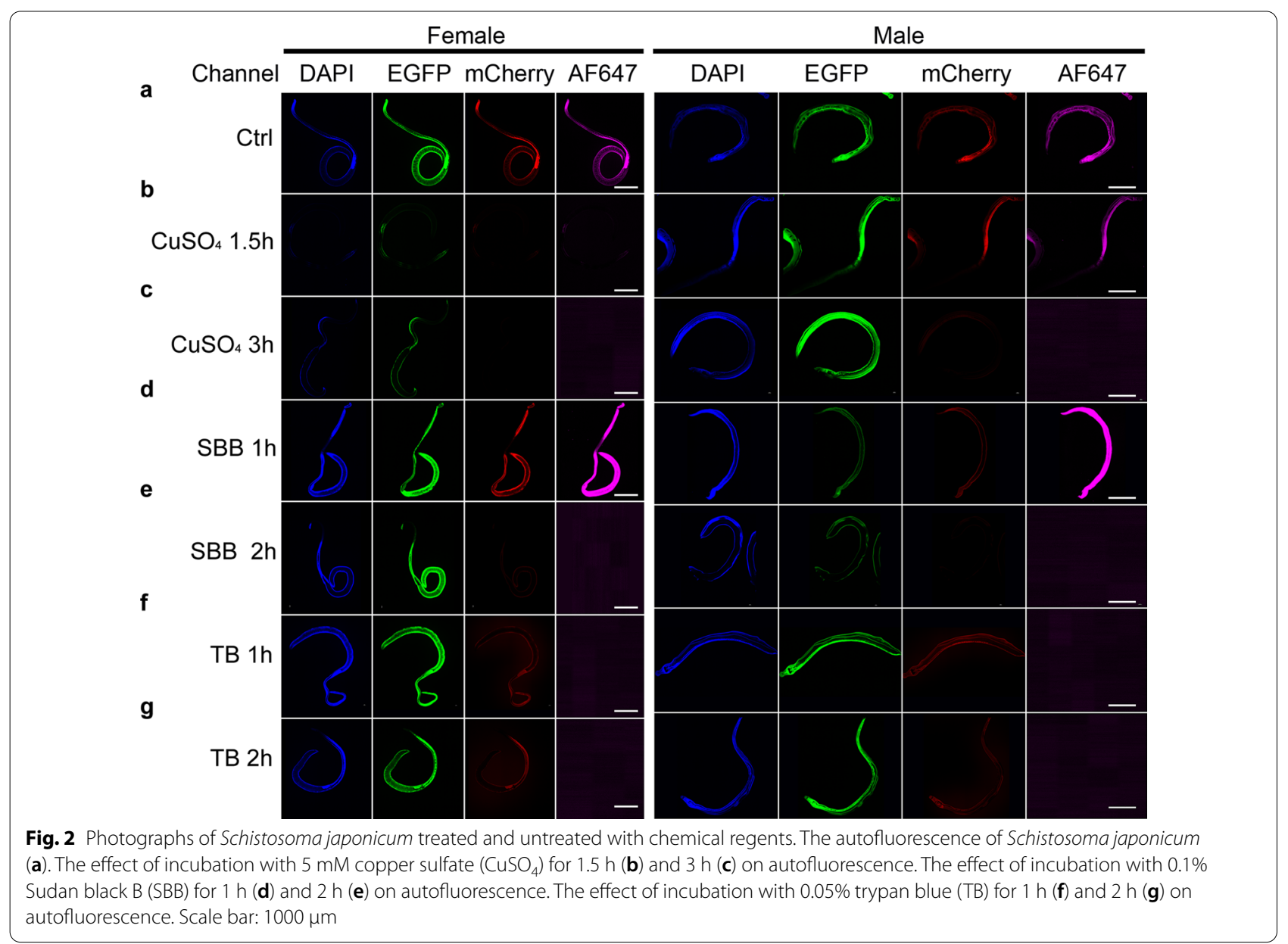

signal did not interfere with the SjCRT signal in males. The IF signals of SjCRT were seen in the anterior end of the esophagus and tegument of female worms ( $i$ and ii in Fig. 4c), and in the ventral sucker and dorsal tegument of male worms (iii and iv in Fig. 4c).

Previous studies have shown that both male and female worms of $S$. japonicum can produce AF under different excitation light irradiation $(405,458,476,488$, 514,543 , and $633 \mathrm{~nm}$ ), with the strongest intensity for green AF (488 nm excitation) [12]. Our results (Fig. 1) show that AF could be observed in S. japonicum worms at $405,488,561$, and $640 \mathrm{~nm}$ excitation wavelengths, with the highest AF intensity produced with excitation at a wavelength of $488 \mathrm{~nm}$. It was also shown that the AF of the male and female tegument and the male intestine was detectable in all channels (Fig. 1), but no AF was detected under the DAPI channel in the vitelline glands or eggs of females (Fig. 1a), which may be associated with endogenous fluorescent substances in the vitelline glands and eggs. Common endogenous fluorescent substances in organisms include amino acids, structural proteins, vitamins, lipopigments, flavins, and porphyrins [23]. During the development of the vitelline gland of schistosome females, three types of inclusions accumulate in the vitelline cells: shell globule clusters, lipid droplets, and $\beta$-glycogen particles [24]. However, the co-localization results for AF and lipid droplet staining of vitelline cells showed that AF did not derive from lipid droplets [14].

The AF features of schistosomes have been applied for diagnosis and vitelline cell separation $[13,14]$, but AF is mainly an interfering signal in IFA for schistosome molecules. In previous studies, IFA of schistosome molecular localization showed that a stronger green AF noise signal was seen in the female gonad and cecum [25] and the eggshell [26, 27], and a weaker red AF signal was observed in female adult schistosomes [28, 29]. In our results, AF was detected in female and male worms in four different channels (Fig. 1). All cases of AF mentioned above suggest that AF reduction is important for the IFA of schistosome molecules. Our assays revealed that $\mathrm{CuSO} 4, \mathrm{SBB}$, and TB had various effects (Fig. 2) on $\mathrm{AF}$ reduction in S. japonicum: $50 \mathrm{mM} \mathrm{CuSO} 4$ reduced $\mathrm{AF}$ in females and $0.5 \% \mathrm{SBB}$ reduced $\mathrm{AF}$ in males (Fig. 3). 


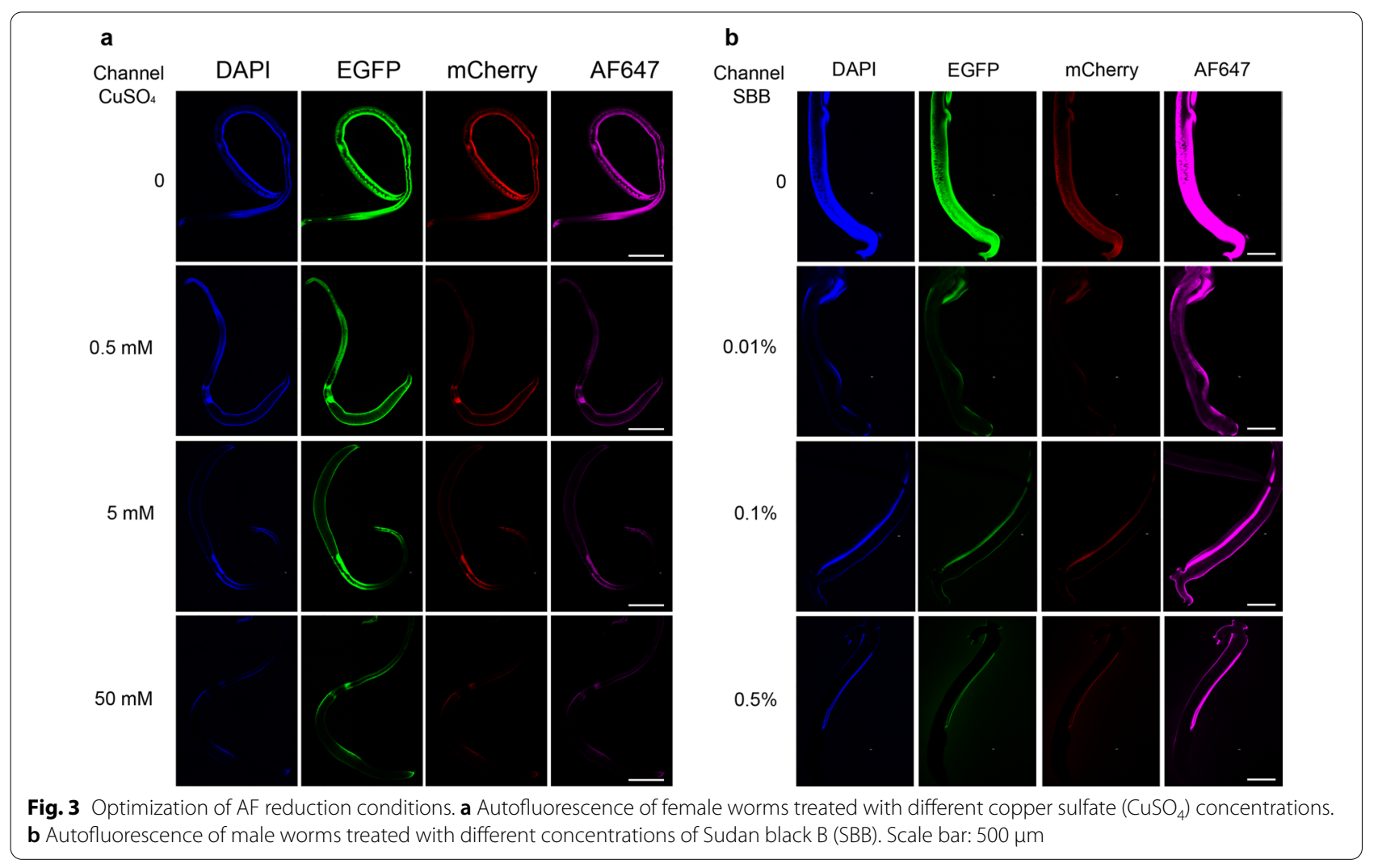

$\mathrm{CuSO}_{4}$ and SBB are widely used chemical reagents to remove AF. In fact, the AF reduction effect of both $\mathrm{CuSO}_{4}$ and SBB can be seen in samples with lipofuscin-like AF [30]. Previous studies have applied $\mathrm{CuSO}_{4}$ to reduce AF of schistosomes for fluorescence in situ hybridization (FISH) [19]. In our study, both $\mathrm{CuSO}_{4}$ and SBB were found to be effective in AF reduction for both female and male worms. However, $\mathrm{CuSO}_{4}$ was more effective for females, while SBB was more effective for males (Fig. 2), although the underlying mechanism of $\mathrm{CuSO}_{4}$ and $\mathrm{SBB}$ in $\mathrm{AF}$ reduction is not well understood. This difference in $\mathrm{AF}$ reduction between males and females still suggests that the reasons for the formation of AF in females and males may not be exactly the same. Triglycerides (TG) have been reported as a lipid associated with AF [31], and there is a significant difference in the composition of triglycerides between male and female schistosomes, with TG (52:1) being more abundant in females, while TG (58:6) is more abundant in males [32]. In addition, a significant difference was found in the uptake of the fluorescent substance hemoglobin between male and female schistosomes [33].

The application of non-fluorescent substrate chromogenic methods (such as HRP/alkaline phosphatase [AP]-labeled secondary antibodies) for localized signal amplification of schistosome proteins can effectively avoid the interference from AF on localized signals. However, non-fluorescence methods will also suffer from interference by the red blood cell products in the worm gut [34]. In a previous study, the localized signal of schistosome proteins was amplified using fluorophore-labeled secondary antibody in IFA. However, due to the lack of an effective AF reduction method used in the assay, a diffuse background signal was present and interfered with positive fluorescence signal recognition [35]. To increase our understanding of the phenomenon and characteristics of schistosome AF, some researchers have labeled the AF and immunofluorescence signals in schistosome IFA based on previous experience [25]. However, experience sometimes limits the accuracy of true positive signal detection, especially when the immunofluorescence signal overlaps with the AF signal. In our study, through reagent screening and condition optimization, a suitable method for AF reduction in male and female schistosomes was developed. Combined with the IFA developed previously [15], we established an effective AF reduction-based IFA which significantly reduced the AF background signal (Additional file 3: Figure S3, Fig. 4a and b EGFP column) and highlighted the immunofluorescence signal (Fig. 4).

The accuracy of immunofluorescence localization of schistosome proteins relies on the ability to reduce 


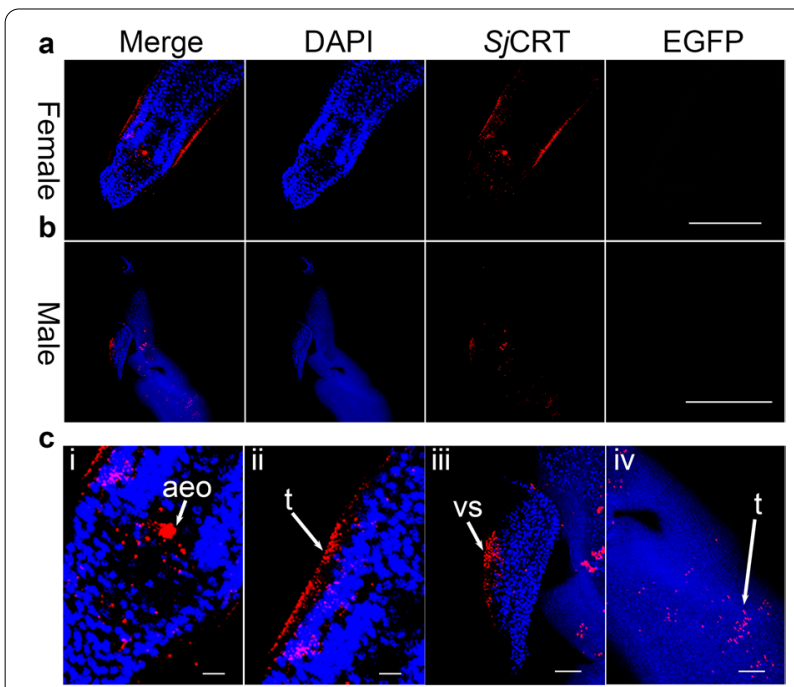

Fig. 4 Reduction of AF for immunofluorescence assay of calreticulin (CRT) in female and male schistosomes. a Immunofluorescence staining for SjCRT in females. Scale bar: $100 \mu \mathrm{m}$. b Immunofluorescence staining for SjCRT in males. Scale bar: $500 \mu \mathrm{m}$. c Merged immunofluorescence images of different body regions of female and male worms; $\mathbf{i}$ the oral sucker and esophagus of female, scale bar: $10 \mu \mathrm{m}$; ii the tegument of female, scale bar: $10 \mu \mathrm{m}$; iii the ventral sucker of male, scale bar: $50 \mu \mathrm{m}$; iv the dorsal tegument of male worms, scale bar: $50 \mu \mathrm{m}$. "Merge" represents the merged images of DAPI/EGFP/SjCRT. DAPI column: staining for the nuclei (blue). EGFP column: no staining, just as a control for detecting AF. SjCRT column: an immunostaining for SjCRT (red). Abbreviations: aeo, the anterior end of the esophagus; $t$, tegument; vs, ventral sucker

noise from AF and improve the specificity of the antigen-antibody reaction. In this study, the use of reagent screening and condition optimization (Figs. 2, 3, and Additional file 1: Figure S1) enabled us to develop a suitable method for AF reduction in both male and female schistosomes. Applied in IFA, AF was effectively reduced (Fig. 4 and Additional file 3: Figure S3). Of course, our attention should also be focused on the specificity of antibodies when immunofluorescence localization is performed on one specific protein. In this study, the specific reaction of anti-SjCRT antibody was verified using the WB method (Additional file 2: Figure S2). The results showed the presence of a single band, the weight mass conformed to the theoretical calculated value, and it was consistent with a previous study [36]. SjCRT is one of the immunostimulatory molecules found to induce a $\mathrm{T}$ helper type 1 (Th1)-polarized immune response in mice [36], and the peptide sequences of SjCRT were detected in excretory/secretory products of $S$. japonicum by proteomics [37]. Therefore, S. japonicum in the vein of mice could regulate the polarization of Th1 cells by excreting SjCRT. The main excretory organs of schistosomes are the tegument and anterior esophageal region [38, 39], and previous omics studies have shown that SjCRT is expressed in the tegument [40]. In this study, SjCRT was observed in the tegument of male and female worms as well as in the anterior end of the esophagus of female worms by IFA (Fig. 4). Our results further confirm that $\mathrm{SjCRT}$ is a protein produced by the excretory organs of S. japonicum.

In this study, only mature S. japonicum male and female worms were used. However, AF has been detected in different kinds of flukes. For example, AF was seen in the vitelline glands [14, 41] and eggs [42, 43] of S. mansoni and Schistosoma haematobium, in the eggs of Clonorchis sinensis [44, 45] and Opisthorchis viverrini [46], and in the vitelline glands of Fasciola gigantica $[47,48]$. In one study, to minimize AF, 15-dayold C. sinensis flukes exhibiting no visible background fluorescence were used for the IFA [49]. However, for samples with AF, increasing the signal-to-noise ratio enables accurate localization of the target molecules. Our method, by reducing AF noise, facilitates improved signal-to-noise ratio and can perhaps be applied to IFA in other flukes.

In summary, both female and male schistosomes have autofluorescence (AF), but the methods for effective AF reduction are different. Our results suggest that $50 \mathrm{mM}$ $\mathrm{CuSO}_{4}$ reduces AF in females and 0.5\% SBB reduces AF in males. The application of this method in IFA of schistosomes can obviously reduce AF and highlight the IF signal. Therefore, our method can improve the accuracy of functional localization for schistosome proteins and provides a foundation for IFA in other flukes.

\section{Abbreviations}

SBB: Sudan black B; TB: Trypan blue; CuSO ${ }_{4}$ : Copper sulfate; Gly: Tris-glycine; AE: Ammonia/ethanol; TG: Triglycerides; SjCRT: Schistosoma japonicum calreticulin; FISH: Fluorescence in situ hybridization; HRP/AP: Horseradish peroxidase or alkaline phosphatase; DAPI: 4',6-Diamidino-2-phenylindole; Cy3: Cyanine 3; AF647: Alexa Fluor 647; AF: Autofluorescence; IF: Immunofluorescence; IFA: Immunofluorescence assay.

\section{Supplementary Information}

The online version contains supplementary material available at https://doi. org/10.1186/s13071-021-05027-3.

Additional file 1: Figure S1. Autofluorescence of different channels of female and male schistosomes treated with Tris-glycine (Gly) or ammonia/ ethanol (AE). Scale bar: $1000 \mu \mathrm{m}$.

Additional file 2: Figure S2. Western blotting result of calreticulin (CRT) in Schistosoma japonicum. Lane 1: protein molecular mass ladder; lane 2: protein extracted from female worms; lane 3: protein extracted from male worms.

Additional file 3: Figure S3. Immunofluorescence control group of female and male worms without anti-SjCRT antibody treatment. Scale bar: $1000 \mu \mathrm{m}$. 


\section{Acknowledgements}

The authors would like to thank Jiange Yang at the State Key Laboratory of Genetic Engineering, Fudan University, for guidance in the immunofluorescence localization experiment.

\section{Authors' contributions}

Manuscript written and data obtained by Q.W. and W.H. Proofreading and literature review done by Z.F, Q.W., and W.H. All authors read and approved the final manuscript.

\section{Funding}

This work was supported by the National Natural Science Foundation of China (No. 31725025) and Shanghai Municipal Science and Technology Committee of Shanghai outstanding academic leaders plan (No. 18XD1400400).

\section{Availability of data and materials}

All materials and data supporting these findings are contained within the manuscript and additional files 1,2,3.

\section{Declarations}

\section{Ethics approval and consent to participate}

Animal procedures were approved by the Ethics and Animal Welfare Committee of the National Institute of Parasitic Diseases, Chinese Center for Disease Control and Prevention, Shanghai, China, (Approval Number: 2013-05). All animal experiments were conducted in accordance with the Guidelines for the Care and Use of Laboratory Animals of the Ministry of Science and Technology of the People's Republic of China, ([2006]398).

\section{Consent for publication}

Not applicable.

\section{Competing interests}

The authors declare no competing financial interests.

\section{Author details}

'State Key Laboratory of Genetic Engineering, Ministry of Education Key Laboratory of Contemporary Anthropology, Collaborative Innovation Center for Genetics and Development, School of Life Sciences, Fudan University, Shanghai 200438, People's Republic of China. ${ }^{2}$ National Institute of Parasitic Diseases, Chinese Center for Disease Control and Prevention, Key Laboratory of Parasite and Vector Biology of the Chinese Ministry of Health, WHO Collaborating Center for Tropical Diseases, Joint Research Laboratory of Genetics and Ecology On Parasite-Host Interaction, Chinese Center for Disease Control and Prevention \& Fudan University, Shanghai 200025, People's Republic of China.

Received: 6 July 2021 Accepted: 18 September 2021

Published online: 14 October 2021

\section{References}

1. McManus DP, Dunne DW, Sacko M, Utzinger J, Vennervald BJ, Zhou X-N. Schistosomiasis. Nat Rev Dis Primers. 2018;4:13.

2. Rashid R, Gaglia G, Chen Y-A, Lin J-R, Du Z, Maliga Z, et al. Highly multiplexed immunofluorescence images and single-cell data of immune markers in tonsil and lung cancer. Sci Data. 2019;6:323.

3. Kann AP, Krauss RS. Multiplexed RNAscope and immunofluorescence on whole-mount skeletal myofibers and their associated stem cells. Development. 2019;146:dev179259.

4. Collins JJ 3rd, Wang B, Lambrus BG, Tharp ME, Iyer H, Newmark PA. Adult somatic stem cells in the human parasite Schistosoma mansoni. Nature. 2013:494:476-9.

5. Wendt GR, Collins JN, Pei J, Pearson MS, Bennett HM, Loukas A, et al. Flatworm-specific transcriptional regulators promote the specification of tegumental progenitors in Schistosoma mansoni. Elife. 2018;7:e33221.

6. El Ridi R, Tallima H. Immunofluorescent localization of proteins in Schistosoma mansoni. Methods Mol Biol. 2020;2151:27-41.
7. Viegas MS, Martins TC, Seco F, do Carmo A. An improved and costeffective methodology for the reduction of autofluorescence in direct immunofluorescence studies on formalin-fixed paraffin-embedded tissues. Eur J Histochem. 2007;51:59-66.

8. Carter SD, Mageswaran SK, Farino ZJ, Mamede Jl, Oikonomou CM, Hope $\mathrm{TJ}$, et al. Distinguishing signal from autofluorescence in cryogenic correlated light and electron microscopy of mammalian cells. J Struct Biol. 2018;201:15-25.

9. Yang $Y$, Honaramooz A. Characterization and quenching of autofluorescence in piglet testis tissue and cells. Anat Res Int. 2012;2012:820120.

10. Jun YW, Kim HR, Reo YJ, Dai M, Ahn KH. Addressing the autofluorescence issue in deep tissue imaging by two-photon microscopy: the significance of far-red emitting dyes. Chem Sci. 2017;8:7696-704.

11. Forge TA, Macguidwin AE. Nematode autofluorescence and its use as an indicator of viability. J Nematol. 1989;21:399-403.

12. Li W, BingYan L, HaiLin D, Xia Z, Wei G, HongXiang Z. Study on autofluorescence and confocal $\lambda$ scanning characteristics of adult Schistosoma japonicum. Chin J Zoonoses. 2009;25:326-9.

13. Domingo M, Mais RF, Weiskopf R, Fink S. Detection of schistosome ova by dark field fluorescence microscopy. Gastroenterology. 1968;54:884-6.

14. Lu Z, Quack T, Hahnel S, Gelmedin V, Pouokam E, Diener M, et al. Isolation, enrichment and primary characterisation of vitelline cells from Schistosoma mansoni obtained by the organ isolation method. Int J Parasitol. 2015:45:663-72.

15. Collins JJ III, King RS, Cogswell A, Williams DL, Newmark PA. An atlas for Schistosoma mansoni organs and life-cycle stages using cell type-specific markers and confocal microscopy. PLoS Negl Trop Dis. 2011;5:e1009.

16. Sun Y, Yu H, Zheng D, Cao Q, Wang Y, Harris D, et al. Sudan black B reduces autofluorescence in murine renal tissue. Arch Pathol Lab Med. 2011;135:1335-42.

17. Baschong W, Suetterlin R, Laeng RH. Control of autofluorescence of archival formaldehyde-fixed, paraffin-embedded tissue in confocal laser scanning microscopy (CLSM). J Histochem Cytochem. 2001;49:1565-72.

18. Bates SR, Tao JQ, Yu KJ, Borok Z, Crandall ED, Collins HL, et al. Expression and biological activity of ABCA1 in alveolar epithelial cells. Am J Respir Cell Mol Biol. 2008;38:283-92.

19. Wang J, Collins JJ 3rd. Identification of new markers for the Schistosoma mansoni vitelline lineage. Int J Parasitol. 2016;46:405-10.

20. Zhu B, Luo F, Shen Y, Yang W, Sun C, Wang J, et al. Schistosoma japonicum cathepsin B2 (SjCB2) facilitates parasite invasion through the skin. PLoS Negl Trop Dis. 2020;14:e0008810.

21. Tarnowski BI, Spinale FG, Nicholson JH. DAPI as a useful stain for nuclear quantitation. Biotech Histochem. 1991;66:297-302.

22. Hossain MZ, Ernst LA, Nagy Jl. Utility of intensely fluorescent cyanine dyes (Cy3) for assay of gap junctional communication by dye-transfer. Neurosci Lett. 1995;184:71-4.

23. Monici M. Cell and tissue autofluorescence research and diagnostic applications. Biotechnol Annu Rev. 2005;11:227-56.

24. Świderski Z, Kacem H, Mackiewicz JS, Miquel J. Functional ultrastructure and cytochemistry of vitellogenesis and mature vitellocytes of the digenean Cainocreadium labracis (Dujardin, 1845), parasite of Dicentrarchus labrax (L., 1758). Parasitol Res. 2019;118:493-504.

25. El-Shehabi F, Ribeiro P. Histamine signalling in Schistosoma mansoni: immunolocalisation and characterisation of a new histamine-responsive receptor (SmGPR-2). Int J Parasitol. 2010;40:1395-406.

26. Edwards J, Brown M, Peak E, Bartholomew B, Nash RJ, Hoffmann KF. The diterpenoid 7-keto-sempervirol, derived from Lycium chinense, displays anthelmintic activity against both Schistosoma mansoni and Fasciola hepatica. PLoS Negl Trop Dis. 2015;9:e0003604.

27. Wang J, Chen R, Collins JJ III. Systematically improved in vitro culture conditions reveal new insights into the reproductive biology of the human parasite Schistosoma mansoni. PLoS Biol. 2019;17:e3000254.

28. He Y, Cai G, Ni Y, Li Y, Zong H, He L. siRNA-mediated knockdown of two tyrosinase genes from Schistosoma japonicum cultured in vitro. Exp Parasitol. 2012;132:394-402.

29. Under E, Huldt G. Antibody-independent binding and activation of complement by Schistosoma mansoni adult worms. Parasite Immunol. 1983;5:183-94. 
30. Schnell SA, Staines WA, Wessendorf MW. Reduction of lipofuscin-like autofluorescence in fluorescently labeled tissue. J Histochem Cytochem. 1999:47:719-30.

31. Meerwaldt R, Lutgers HL, Links TP, Graaff R, Baynes JW, Gans RO, et al. Skin autofluorescence is a strong predictor of cardiac mortality in diabetes. Diabetes Care. 2007;30:107-12.

32. Kadesch P, QuackT, Gerbig S, Grevelding CG, Spengler B. Tissue- and sex-specific lipidomic analysis of Schistosoma mansoni using high-resolution atmospheric pressure scanning microprobe matrix-assisted laser desorption/ionization mass spectrometry imaging. PLoS Negl Trop Dis. 2020;14:e0008145.

33. Xiao SH, Sun J. Schistosoma hemozoin and its possible roles. Int J Parasitol. 2017:47:171-83.

34. You H, Gobert GN, Du X, Pali G, Cai P, Jones MK, et al. Functional characterisation of Schistosoma japonicum acetylcholinesterase. Parasit Vectors. 2016;9:328.

35. Taman A, Ribeiro P. Glutamate-mediated signaling in Schistosoma mansoni: a novel glutamate receptor is expressed in neurons and the female reproductive tract. Mol Biochem Parasitol. 2011;176:42-50.

36. Ma L, Li D, Yuan C, Zhang X, Ta N, Zhao X, et al. SjCRT, a recombinant Schistosoma japonicum calreticulin, induces maturation of dendritic cells and a Th1-polarized immune response in mice. Parasit Vectors. 2017;10:570.

37. Liu F, Cui SJ, Hu W, Feng Z, Wang ZQ, Han ZG. Excretory/secretory proteome of the adult developmental stage of human blood fluke Schistosoma japonicum. Mol Cell Proteomics. 2009;8:1236-51.

38. Faghiri Z, Camargo SM, Huggel K, Forster IC, Ndegwa D, Verrey F, et al. The tegument of the human parasitic worm Schistosoma mansoni as an excretory organ: the surface aquaporin SmAQP is a lactate transporter. PLOS ONE. 2010;5:e10451.

39. Li XH, Stark M, Vance GM, Cao JP, Wilson RA. The anterior esophageal region of Schistosoma japonicum is a secretory organ. Parasit Vectors. 2014;7:565.

40. Liu F, Lu J, Hu W, Wang SY, Cui SJ, Chi M, et al. New perspectives on hostparasite interplay by comparative transcriptomic and proteomic analyses of Schistosoma japonicum. PLoS Pathog. 2006;2:e29.
41. Burden CS, Ubelaker JE. Schistosoma mansoni and Schistosoma haematobium: difference in development. Exp Parasitol. 1981;51:28-34.

42. Schramm G, Suwandi A, Galeev A, Sharma S, Braun J, Claes A-K, et al. Schistosome eggs impair protective Th1/Th17 immune responses against Salmonella infection. Front Immunol. 2018;9:2614.

43. Rinaldi G, Okatcha TI, Popratiloff A, Ayuk MA, Suttiprapa S, Mann VH, et al. Genetic manipulation of Schistosoma haematobium, the neglected schistosome. PLoS Negl Trop Dis. 2011;5:e1348.

44. Kang JM, Sohn WM, Ju JW, Kim TS, Na BK. Identification and characterization of a serine protease inhibitor of Clonorchis sinensis. Acta Trop. 2010;116:134-40.

45. Wang X, Chen W, Hu F, Deng C, Zhou C, Lv X, et al. Clonorchis sinensis enolase: identification and biochemical characterization of a glycolytic enzyme from excretory/secretory products. Mol Biochem Parasitol. 2011;177:135-42.

46. Amornpunt S, Sarasombath S, Sirisinha S. Production and characterization of monoclonal antibodies against the excretory-secretory antigen of the liver fluke (Opisthorchis viverrini). Int J Parasitol. 1991;21:421-8.

47. Anuracpreeda P, Chawengkirttikul R, Sobhon P. Immunodiagnosis of Fasciola gigantica infection using monoclonal antibody-based sandwich ELISA and immunochromatographic assay for detection of circulating cathepsin L1 protease. PLoS ONE. 2016;11:e0145650.

48. Meemon K, Khawsuk W, Sriburee S, Meepool A, Sethadavit M, Sansri V, et al. Fasciola gigantica: histology of the digestive tract and the expression of cathepsin L. Exp Parasitol. 2010;125:371-9.

49. Dai F, Yoo WG, Lu Y, Song JH, Lee JY, Byun Y, et al. Sodium-bile acid cotransporter is crucial for survival of a carcinogenic liver fluke Clonorchis sinensis in the bile. PLoS Negl Trop Dis. 2020;14:e0008952.

\section{Publisher's Note}

Springer Nature remains neutral with regard to jurisdictional claims in published maps and institutional affiliations.
Ready to submit your research? Choose BMC and benefit from:

- fast, convenient online submission

- thorough peer review by experienced researchers in your field

- rapid publication on acceptance

- support for research data, including large and complex data types

- gold Open Access which fosters wider collaboration and increased citations

- maximum visibility for your research: over $100 \mathrm{M}$ website views per year

At BMC, research is always in progress.

Learn more biomedcentral.com/submissions 